\title{
STABILITY AND ROBUSTNESS OF DISTURBED-PORT CONTROLLED HAMILTONIAN SYSTEMS WITH DISSIPATION
}

\author{
M. Becherif* E. Mendes **
}

\author{
* LSS \& LGEP, CNRS-SUPELEC, Gif-sur-Yvette 91192, \\ FRANCE, becherif@lss.supelec.fr \\ ** LCIS, ESISAR 50, rue Barthélémy de Laffémas, BP 54 \\ 26902 Valence Cedex 9, FRANCE, \\ Eduardo.Mendes@esisar.inpg.fr
}

\begin{abstract}
:
A recent approach of Passivity-Based Control (PBC) is the Interconnection and Damping Assignment Passivity-Based Control (IDA-PBC) which is a very useful technique to control systems assigning a desired Port-Controlled Hamiltonian $(\mathrm{PCH})$ structure to the closed-loop. IDA-PBC provides sometimes control laws that are complicated and/or need all state measurement. In this paper, DisturbedPCHD systems are considered where IDA-PBC is applied. Two sufficient stability conditions on the disturbances are given. Moreover, this approach allows to study controller robustness against parameters uncertainties. This new approach simplify the control law and allows to deal with unmeasured terms. Application of this new approach to the control of the voltage-fed Induction Machine (IM) is presented. Copyright@2005 IFAC.
\end{abstract}

Keywords: Nonlinear control, Stability analysis, Robustness, Passive, Induction machines.

\section{INTRODUCTION}

$\mathrm{PCH}^{1}$ systems were introduced by van der Schaft and Maschke in the early nineties van der Schaft and Maschke (1994) and has since grown to become a large field of interest in the research of electrical, mechanical and electro-mechanical systems. Some of the advantages of expressing systems in $\mathrm{PCH}$ form is the fact that they cover a large set of physical systems and capture important structural properties. See van der Schaft (2000) for an in-depth review of PCH systems.

Consider the nonlinear system given by:

$$
\dot{x}=f(x)+g(x) u
$$

\footnotetext{
1 Consider also the Port Controlled Hamiltonian systems with Dissipation (PCHD)
}

where $x \in \mathbb{R}^{n}$ is the state vector, $f(x)$ and $g(x)$ are locally Lipschitz functions and $u \in \mathbb{R}^{m}$ is the control input.

A PCHD form of the system (1) is given by:

$$
\dot{x}=[J(x)-\mathcal{R}(x)] \frac{\partial H}{\partial x}(x)
$$

where $J(x)$ is an $n \times n$ skew symmetric matrix with entries depending smoothly on $x, \mathcal{R}(x)$ is a $n \times n$ positive semi-definite symmetric matrix depending smoothly on $x$ and $\frac{\partial H}{\partial x}$ is the gradient vector of the energy function $H(x)$ of the system (1).

PCH systems, with $H(x)$ non-negative, are passive $^{2}$ systems and therefore inherently stable.

\footnotetext{
2 A passive system is one where the stored energy cannot exceed the energy supplied to it from its environment, the difference being dissipated.
} 
This is usually not good enough from a control engineering point of view, because the system might only be marginally stable or stable around an undesired equilibrium. There are also performances considerations to be dealt with. A recent and very interesting approach to solve these problems is the IDA-PBC method, which is a general way of stabilizing a large class of physical systems, see Ortega et al. (2002).

Using the IDA-PBC approach yields generally to the resolution of partial differential equations that can be very complex or to a complicated closed loop energy function that induces a complex controllers which may need measurement of all the states.

A new approach is proposed in this paper to apply the IDA-PBC technique to closed loop systems having the following Disturbed-PCHD form:

$$
\dot{\tilde{x}}=\left[J_{d}(\tilde{x})-\mathcal{R}_{d}(\tilde{x})\right] \frac{\partial H_{d}}{\partial \tilde{x}}(\tilde{x})+\xi
$$

where $(\tilde{})=()-.(.)_{d}$ is the error signal, $(.)_{d}$ is the reference, $J_{d}(\tilde{x})$ is a desired $n \times n$ skew symmetric matrix with entries depending smoothly on $\tilde{x}$, $\mathcal{R}_{d}(\tilde{x})$ is a desired positive semi-definite symmetric matrix depending smoothly on $\tilde{x}$ and $H_{d}(\tilde{x})$ is the desired closed loop energy function that takes its minimum in the equilibrium coordinates of the system, and $\xi$ is a disturbance.

An expected benefit of such a methodology is that it leads to physically interpretable controllers, which possess inherent robustness properties. In addition, allowing the existence of the disturbance permits to cover a large class of physical system and/or considerably simplify the controller (see next application sections).

The purpose of examples given in this paper are to give different $\mathrm{PBC}$ to the speed control of the IM. Firstly, the IDA-PBC of the IM constitutes a first-if modest-attempt to apply the recent IDAPBC technique to the IM. Secondly, to extend the application of the IDA-PBC technique to the class of nonlinear systems with presence of disturbance in the model and give, in this case, some sufficient conditions to prove the stability. Thirdly, it is shown that the Field Oriented Control (FOC) of the IM is a particular case of the IDA-PBC technique applied to the Disturbed-PCHD structure.

\section{MAIN RESULTS}

\subsection{IDA-PBC of a Disturbed PCHD system}

The aim of this section is to enlarge the application of the existent control techniques (Maschke et al. (1998), Maschke et al. (1999), van der Schaft (2000)) applied to PCHD systems in the presence of disturbance.
Consider the disturbed nonlinear system given by:

$$
\dot{x}=f(x)+g(x) u+\zeta
$$

where $x$ is the state vector, $f(x)$ and $g(x)$ are locally Lipschitz functions and $u \in \mathbb{R}^{m}$ is the control input and $\zeta$ is a disturbance.

The IDA-PBC approach is used to find the control input $u$ that renders (4) in closed loop written in the Disturbed-PCHD form (3). In (3), $\xi$ catch unmeasured and/or complex terms that are not welcome in the control law, also parameters uncertainties can be putted in $\xi$. Note that $\xi$ can be equal to $\zeta$.

The two following main theorems state sufficient conditions to ensure global stability of a Disturbed-PCHD system equilibrium point.

Theorem 1. Consider the closed loop dynamic (3) and $\dot{H}_{d}=\nabla H_{d}^{T} \dot{\tilde{x}}$ the time derivative of the nonnegative energy function $H_{d}$ along the trajectories of (3). Let $\kappa$ be a positive constant. Let $\lambda_{\min }\left(R_{d}(\tilde{x})\right)$ be the smallest eigenvalue of $R_{d}(\tilde{x})$. Let denote the euclidian norm $|\cdot|$.

If

$$
|\xi|<\kappa\left|\nabla H_{d}\right|, \kappa<\lambda_{\min }\left(\mathcal{R}_{d}(\tilde{x})\right),
$$

Then

$$
\dot{H}_{d} \leq 0
$$

and the equilibrium $\tilde{x}=0$ of (3) is globally stable.

Furthermore, if $\left.\dot{H}_{d}(\tilde{x})\right|_{\tilde{x}=0} \Rightarrow \tilde{x}=0$ then the equilibrium $\tilde{x}=0$ of (3) is globally asymptotically stable.

$$
\begin{aligned}
& \text { Proof. } \\
& \qquad \begin{aligned}
\dot{H}_{d} & =\nabla H_{d}^{T} \dot{\tilde{x}} \\
& =\nabla H_{d}^{T}\left[J_{d}(\tilde{x})-R_{d}(\tilde{x})\right] \nabla H_{d}+\nabla H_{d}^{T} \xi \\
& =-\nabla H_{d}^{T} R_{d}(\tilde{x}) \nabla H_{d}+\nabla^{T} H_{d} \xi
\end{aligned}
\end{aligned}
$$

If $|\xi|<\lambda_{\min }\left(R_{d}(\tilde{x})\right)\left|\nabla H_{d}\right|$

$$
\begin{gathered}
\Rightarrow \dot{H}_{d} \leq-\nabla H_{d}^{T}\left[\left|R_{d}(\tilde{x})\right|-\lambda_{\min }\left(R_{d}(\tilde{x})\right)\right] \nabla H_{d} \leq 0 \\
\text { where } \quad\left|R_{d}(\tilde{x})\right| \geq \lambda_{\min }\left(R_{d}(\tilde{x})\right)
\end{gathered}
$$

Theorem 2. Consider the closed loop dynamic (3). Let $A(\tilde{x})$ be a $n \times n$ matrix such that

$$
\xi=A(\tilde{x}) \nabla H_{d}
$$

If $\left[R_{d}(\tilde{x})-A(\tilde{x})\right]$ is non-negative definite, then the equilibrium $\tilde{x}=0$ of (3) is globally stable.

Furthermore, if $\left.\dot{H}_{d}(\tilde{x})\right|_{\tilde{x}=0} \Rightarrow \tilde{x}=0$ then the equilibrium $\tilde{x}=0$ of (3) is globally asymptotically stable.

$$
\begin{aligned}
& \text { Proof. } \\
& \dot{H}_{d}=-\nabla H_{d}^{T} R_{d}(\tilde{x}) \nabla H_{d}+\nabla H_{d}^{T} \xi \\
& =-\nabla H_{d}^{T}\left[R_{d}(\tilde{x})-A(\tilde{x})\right] \nabla H_{d} \\
& \dot{H}_{d} \leq 0 \Leftrightarrow\left[R_{d}(\tilde{x})-A(\tilde{x})\right] \geq 0 \text {. }
\end{aligned}
$$




\subsection{Robustness analysis}

The main expected benefit of the proposed IDAPBC of Disturbed-PCHD systems is the design of robust controllers. For example, assume the existence of parameters uncertainties in model (3). The uncertainties can be represented by $\Delta \varepsilon$ as:

$$
\begin{gathered}
\dot{\tilde{x}}=\left[J_{d}(\tilde{x})-\mathcal{R}_{d}(\tilde{x})+\Delta \varepsilon\right] \frac{\partial H_{d}}{\partial \tilde{x}}(\tilde{x})+\xi \\
\Leftrightarrow \dot{\tilde{x}}=\left[J_{d}(\tilde{x})-\mathcal{R}_{d}(\tilde{x})\right] \frac{\partial H_{d}}{\partial \tilde{x}}(\tilde{x})+\underbrace{\xi+\Delta \varepsilon \frac{\partial H_{d}}{\partial \tilde{x}}(\tilde{x})}_{\psi}
\end{gathered}
$$

where $\psi$ plays the role of the new disturbance and the control synthesis procedure can be applied on system (11) allowing then the design of a robust controller toward parameters uncertainties.

To illustrate the usefulness of theorems 1 and 2, next sections present different applications of the IDA-PBC of the mechanical speed and rotor flux norm control of a voltage-fed IM. The connection of the IDA-PBC control and the classical FOC is shown.

\section{DYNAMIC MODEL OF THE IM}

\section{$3.1 \alpha \beta$ model}

The standard two phase $\alpha \beta$-model ${ }^{3}$ of an $n_{p}$ pole pair squirrel cage IM with uniform air-gap can be written as:

$\Sigma_{e}$ is the electrical subsystem.

$$
\begin{gathered}
\lambda=L(\theta) i \\
\dot{\lambda}+R i=v
\end{gathered}
$$

$\Sigma_{m}$ is the mechanical subsystem.

$$
\begin{array}{r}
j \dot{\omega}=\tau-\tau_{L} \\
\tau=\frac{1}{2} i^{T} \frac{\partial L(\theta)}{\partial \theta} i
\end{array}
$$

with electrical parameters:

$$
L(\theta)=\left[\begin{array}{cc}
L_{s} I_{2} & L_{s r} e^{J n_{p} \theta} \\
L_{s r} e^{-J n_{p} \theta} & L_{r} I_{2}
\end{array}\right] \in \mathbb{R}^{4 x 4}
$$

where $L(\theta)$ is the inductance matrix, $r, s$ denotes the rotor and stator, respect., $L_{s r}$ denotes the mutuel inductance, $0<\sigma=1-\frac{L_{s r}^{2}}{L_{s} L_{r}}<1$

$$
\begin{gathered}
e^{J n_{p} \theta}=\left[\begin{array}{cc}
\cos \left(n_{p} \theta\right) & -\sin \left(n_{p} \theta\right) \\
\sin \left(n_{p} \theta\right) & \cos \left(n_{p} \theta\right)
\end{array}\right], J=\left[\begin{array}{cc}
0 & -1 \\
1 & 0
\end{array}\right], \\
R=\operatorname{diag}\left\{R_{s} I_{2}, R_{r} I_{2}\right\}
\end{gathered}
$$

$R_{s}, R_{r}>0$ are stator and rotor resistances. $I_{2}$ is the $2 \times 2$ identity matrix. $\theta$ is the mechanical angle, $\tau_{L}$ is the load torque.

$$
\lambda=\left[\begin{array}{ll}
\lambda_{s} & \lambda_{r}
\end{array}\right]^{T}, i=\left[\begin{array}{ll}
i_{s} & i_{r}
\end{array}\right]^{T}, v=\left[\begin{array}{ll}
v_{s} & 0
\end{array}\right]^{T}
$$

3 In this model the axes for the stator have a fixed position while those corresponding to the rotor are rotating at the rotor (electrical) angular speed

\section{2 dq-model of the IM}

The model in the classical $d q$ frame is:

$$
\begin{gathered}
{\left[\begin{array}{c}
\dot{i}_{d q} \\
\dot{\lambda}_{d q} \\
j \dot{\omega}
\end{array}\right]=\left[\begin{array}{cc}
-\left[J\left(\dot{\rho}+n_{p} \dot{\theta}\right)+\gamma I_{2}\right] & \frac{L_{s r}}{T_{r}} \frac{1}{\sigma L_{s} L_{r}} I_{2} \\
\frac{L_{s r}}{T_{r}} I_{2} & -\left[\begin{array}{c}
\dot{\rho} J+\frac{1}{T_{r}} I_{2}
\end{array}\right] \\
\frac{n_{p} L_{s r}}{L_{r}} \lambda_{d q}^{T} J & 0 \\
& -\frac{n_{p} L_{s r}}{\sigma L_{s} L_{r}} J \lambda_{d q} \\
0 \\
0
\end{array}\right]\left[\begin{array}{c}
i_{d q} \\
\lambda_{d q} \\
\omega
\end{array}\right]+\left[\begin{array}{c}
\frac{1}{\sigma L_{s}} u_{d q} \\
0 \\
-\tau_{L}
\end{array}\right]}
\end{gathered}
$$

where $\dot{\rho}=\frac{R_{r}}{n_{p} \beta^{2}} \frac{n_{p} L_{s r}}{L_{r}}\left(\lambda_{d} i_{q}-\lambda_{q} i_{d}\right)=\frac{L_{s r}}{T_{r}} \frac{i_{q}}{\lambda_{d}}(19)$

\subsection{Geometric properties and zero dynamics}

It is shown in Ortega et al. (1998) that IM is "invertible" and the zero dynamics of the motor with outputs $\tau$ and $\left|\lambda_{r}\right|$ are periodic. Then, if $\tau$ and $\left|\lambda_{r}\right|$ are fixed to constant values, the rotor flux rotates at a constant speed. This expression also shows that torque can be controlled by controlling rotor flux norm and slip speed -as is well known in the drives community.

\section{PROBLEM FORMULATION}

The control aim is the mechanical speed regulation with a constant rotor flux norm. Then, the system output variable is $y=\omega$ and the control objectives are:

$$
\lim _{t \mapsto+\infty} y=y^{d}, \quad \lim _{t \mapsto+\infty}\left|\lambda_{r}\right|=\left|\lambda_{r}^{d}\right|=\beta_{*}
$$

with $\quad \lambda_{r}^{d}=e^{J \rho_{d}}\left[\begin{array}{c}\beta_{*} \\ 0\end{array}\right], \quad \dot{\rho}_{d}=\frac{R_{r}}{n_{p} \beta_{*}^{2}} \tau_{d}$

then $\dot{\lambda}_{r}^{d}=\frac{R_{r}}{n_{p} \beta_{*}^{2}} \tau_{d} J \lambda_{r}^{d}$. $\tau_{d}$ is the desired torque, which is defined via a PI velocity loop as:

$$
\begin{gathered}
\tau_{d}=-K_{p_{e x t}} \tilde{y}-z \\
\dot{z}=K_{i_{e x t}} \tilde{y}
\end{gathered}
$$

with $K_{p_{e x t}}, K_{i_{e x t}}>0$.

In the next sections the speed control of the IM will be solved by different IDA-PBC controllers applied to a Disturbed-PCHD systems, such that: 1- IDA-PBC of IM while all states are measurables.

2- IDA-PBC of IM feeding back stator currents only.

Remark 1. It can be seen that for the speed control of the IM, the rotor flux rotates at a constant speed. Thus, we have an equilibrium orbit. To apply the IDA-PBC technique it is needed to have an equilibrium points. To overcome this problem, two solutions can be adopted:

- Make a change of coordinates to get in a frame 
where the equilibrium orbit becomes an equilibrium points.

- Express the model as an error dynamics.

Remark 2. The IM can be decomposed as a feedback interconnection of an electrical subsystem $\Sigma_{e}$ $: u \rightarrow[i \lambda]^{T}$, and a mechanical subsystem $\Sigma_{m}$ $:\left(\omega-\omega_{d}\right) \rightarrow \tau_{d}$. Since $\Sigma_{m}$ can be controlled with just a classical linear controller it is reasonable to concentrate our attention on the problem of controlling $\Sigma_{e}$. An additional motivation for this approach stems from the fact that typically there is a natural time-scale decomposition between the electrical and mechanical dynamics. The reader is referred to Ortega et al. (1998) for motivation and additional details on this control approach.

\section{IDA-PBC OF VOLTAGE-FED IM WITH ALL STATES MEASURABLES}

The control of the electrical subsystem $\Sigma_{e}$ is first considered. From (18) $\Sigma_{e}$ is:

$$
\begin{aligned}
{\left[\begin{array}{c}
\dot{i}_{d q} \\
\dot{\lambda}_{d q}
\end{array}\right]=} & {\left[\begin{array}{cc}
-\left[J\left(\dot{\rho}+n_{p} \dot{\theta}\right)+\gamma I_{2}\right] & -\frac{L_{s r}}{\sigma L_{s} L_{r}}\left[J n_{p} \dot{\theta}-\frac{1}{T_{r}} I_{2}\right] \\
\frac{L_{s r}}{T_{r}} I_{2} & -\left(\dot{\rho} J+\frac{1}{T_{r}} I_{2}\right)
\end{array}\right] } \\
& \times\left[\begin{array}{c}
i_{d q} \\
\lambda_{d q}
\end{array}\right]+\left[\begin{array}{c}
\frac{1}{\sigma L_{s}} u_{d q} \\
0
\end{array}\right]
\end{aligned}
$$

Consider the stored energy as follows:

$$
H=\frac{1}{2} \tilde{i}_{d q}^{2}+\frac{1}{2} \tilde{\lambda}_{d q}^{2} \quad \Rightarrow \nabla H=\left[\begin{array}{l}
\tilde{i}_{d q} \\
\tilde{\lambda}_{d q}
\end{array}\right]
$$

with $\nabla H=\left[\begin{array}{c}\nabla_{i_{d q}} H \\ \nabla_{\lambda_{d q}} H\end{array}\right],\left[\begin{array}{c}i_{d q}^{*} \\ \lambda_{d q}^{*}\end{array}\right]=\operatorname{argmin}(H)$

where $(\tilde{.})=()-.(.)^{*}$.

The purpose is to obtain in closed loop the following Disturbed-PCHD form:

$$
\left[\begin{array}{l}
\dot{\tilde{i}}_{d q} \\
\dot{\tilde{\lambda}}_{d q}
\end{array}\right] \equiv\left[\begin{array}{cc}
J_{11}-R_{1} & J_{12} \\
-J_{12}^{T} & J_{22}-R_{2}
\end{array}\right] \nabla H+\xi
$$

with $\xi$ a disturbance of the form ${ }^{4}$ :

$$
\xi=\left[\begin{array}{cc}
0 & J_{a b} \\
0 & 0
\end{array}\right] \nabla H \triangleq A(\tilde{x}) \nabla H
$$

$J_{i i}=-J_{i i}^{T}, R_{i}=R_{i}^{T}>0, J_{a b}$ is a $2 \times 2$ matrix to be defined

\subsection{IDA-PBC procedure}

Consider equilibrium point of the two lower equation of $(24)$ :

$$
\dot{\lambda}_{d q}^{*}=\frac{L_{s r}}{T_{r}} i_{d q}^{*}-\left(\dot{\rho} J+\frac{1}{T_{r}} I_{2}\right) \lambda_{d q}^{*}
$$

4 Here the choice of $\xi$ is motivated by removing terms destroying the properties of $\left[\mathcal{J}_{d}-\mathcal{R}_{d}\right]$ given in theorem 1 .
Subtract (26) from the last two equations of (24) (unactuated coordinate) and identify with (25):

$\frac{L_{s r}}{T_{r}} \tilde{i}_{d q}-\left(\dot{\rho} J+\frac{1}{T_{r}} I_{2}\right) \tilde{\lambda}_{d q} \equiv-J_{12}^{T} \tilde{i}_{d q}+\left(J_{22}-R_{2}\right) \tilde{\lambda}_{d q}$

One solution is to take:

$$
\left\{\begin{array}{c}
J_{12}^{T}=-\frac{L_{s r}}{T_{r}} I_{2} \\
J_{22}=-\dot{\rho} J \\
R_{2}=\frac{1}{T_{r}} I_{2}>0
\end{array}\right.
$$

Substrat $\frac{d}{d t} i_{d q}^{*}$ from the two upper equation of (24). Actuated equation of (24):

$$
\begin{aligned}
& -\frac{d}{d t} i_{d q}^{*}-\left[J\left(\dot{\rho}+n_{p} \dot{\theta}\right)+\gamma I_{2}\right] i_{d q}-\frac{L_{s r}}{\sigma L_{s} L_{r}}\left[J n_{p} \dot{\theta}-\frac{1}{T_{r}} I_{2}\right] \lambda_{d q} \\
& +\frac{1}{\sigma L_{s}} u_{d q} \equiv\left(J_{11}-R_{1}\right) \tilde{i}_{d q}+\left(J_{12}+J_{a b}\right) \tilde{\lambda}_{d q}
\end{aligned}
$$

Let take $J_{a b}=-J_{12}>0$ and $J_{11}=0$. Thus,

$-\frac{d}{d t} i_{d q}^{*}-\left[J\left(\dot{\rho}+n_{p} \dot{\theta}\right)+\gamma I_{2}\right] i_{d q}-\frac{L_{s r}}{\sigma L_{s} L_{r}}\left[J n_{p} \dot{\theta}-\frac{1}{T_{r}} I_{2}\right] \lambda_{d q}$ $+\frac{1}{\sigma L_{s}} u_{d q} \equiv-R_{1} \tilde{i}_{d q}$

Then with the following control:

$$
\begin{aligned}
u_{d q} & =\sigma L_{s}\left\{\frac{d}{d t} i_{d q}^{*}+\left[J\left(\dot{\rho}+n_{p} \dot{\theta}\right)+\gamma I_{2}\right] i_{d q}+\right. \\
& \left.+\frac{L_{s r}}{\sigma L_{s} L_{r}}\left[J n_{p} \dot{\theta}-\frac{1}{T_{r}} I_{2}\right] \lambda_{d q}-R_{1} \tilde{i}_{d q}\right\}
\end{aligned}
$$

(24) can be written in Disturbed-PCHD form as

$$
\begin{aligned}
& {\left[\begin{array}{c}
\dot{\tilde{i}}_{d q} \\
\dot{\tilde{\lambda}}_{d q}
\end{array}\right]=\left[\begin{array}{cc}
-R_{1} & -\frac{L_{s r}}{T_{r}} I_{2} \\
\frac{L_{s r}}{T_{r}} I_{2} & -\dot{\rho} J-\frac{1}{T_{r}} I_{2}
\end{array}\right] \nabla H+\left[\begin{array}{cc}
0 & \frac{L_{s r}}{T_{r}} I_{2} \\
0 & 0
\end{array}\right] \nabla H(29)} \\
& =\left[\begin{array}{cc}
J_{11}-R_{1} & J_{12} \\
-J_{12}^{T} & J_{22}-R_{2}
\end{array}\right] \nabla H+\xi \\
& \text { with } J_{11}=0, \quad J_{12}^{T}=-\frac{L_{s r}}{T_{r}} I_{2}, \quad J_{22}=-\dot{\rho} J, \\
& \text { and } R_{2}=\frac{1}{T_{r}} I_{2}>0, \quad J_{a b}=-J_{12} .
\end{aligned}
$$

Theorem 3. Consider the electrical subsystem (24) with $\dot{\rho}$ given by (19). Let all state vector be measured. Let the control input $u_{d q}$ be (28). Then,

System (24) can be written, in closed loop, in the Disturbed-PCHD form (29) and the equilibrium $\left[\begin{array}{ll}\tilde{i}_{d q} & \tilde{\lambda}_{d q}\end{array}\right]^{T}=0$ of (29) is globally asymptotically stable if $R_{1}>\frac{L_{s r}^{2}}{4 T_{r}} I_{2}$, with $R_{1}$ a design parameter.

Proof. Consider (29) with control (28), applying theorem 2 gives:

$$
Q(\tilde{x}) \triangleq\left[\mathcal{R}_{d}(\tilde{x})-A(\tilde{x})\right]=\left[\begin{array}{cc}
R_{1} & J_{a b} \\
0 & R_{2}
\end{array}\right]
$$

$Q(\tilde{x})$ needs to be non negative definite. 
$X^{T} Q(\tilde{x}) X=X^{T}\left[\begin{array}{cc}R_{1} & J_{a b} \\ 0 & R_{2}\end{array}\right] X=X^{T} \underbrace{\left[\begin{array}{cc}R_{1} & \frac{1}{2} J_{a b} \\ \frac{1}{2} J_{a b}^{T} & R_{2}\end{array}\right]}_{\triangleq Q^{\prime}(\tilde{x})} X$ with $R_{j}=R_{j}^{T}>0, J_{a b}=\frac{L_{s r}}{T_{r}} I_{2}=J_{a b}^{T}, j=1,2$. $Q^{\prime}(\tilde{x}) \geq 0 \Leftrightarrow Q(\tilde{x}) \geq 0$, apply then the Schur's Complement.

$\left.\left.Q^{\prime}(\tilde{x}) \geq 0 \Leftrightarrow i\right) R_{1} \geq 0, i i\right) R_{2}-\frac{1}{4} J_{a b}\left(R_{1}\right)^{-1} J_{a b} \geq 0$

$i)$ is verified.

$i i) \Leftrightarrow R_{1}^{-1} \leq 4 J_{a b}^{-1} R_{2} J_{a b}^{-1} \Leftrightarrow R_{1} \geq \frac{L_{s r}^{2}}{4 T_{r}} I_{2}$

Thus, if $R_{1} \geq \frac{L_{s r}^{2}}{4 T_{0}} I_{2} \Leftrightarrow Q(\tilde{x})$ is non negative definite $\Rightarrow \dot{H} \leq 0$ and the system is globally asymptotically stable.

\subsection{IDA-PBC and Direct FOC control of the IM}

The FOC given in Ortega et al. (1998) is:

$$
\begin{aligned}
u_{d q}^{F O C}= & \sigma L_{s}\left\{\frac{d}{d t} i_{d q}^{*}+\left[J\left(\dot{\rho}+n_{p} \dot{\theta}\right)+\gamma I_{2}\right] i_{d q}+\right. \\
& \left.\frac{L_{s r}}{\sigma L_{s} L_{r}}\left(J n_{p} \dot{\theta}-\frac{1}{T_{r}} I_{2}\right) \lambda_{d q}+v_{d q}^{F O C}\right\}
\end{aligned}
$$

where $\dot{\rho}$ is given by (19). $v_{d q}^{F O C}$ is defined to force $i_{d}$ and $i_{q}$ to their desired values.

$$
v_{d q}^{F O C}=F(p)\left(i_{d q}^{*}-i_{d q}\right)=F(p) \tilde{i}_{d q}
$$

with $F(p)$ is usually a linear controller.

The IDA-PBC technique gives the following control law (with the chosen $\left(\mathcal{J}_{d}(\tilde{x})-\mathcal{R}_{d}\right)$ matrix)

$$
\begin{aligned}
u_{d q}^{I D A}= & \sigma L_{s}\left\{\frac{d}{d t} i_{d q}^{*}+\left[J\left(\dot{\rho}+n_{p} \dot{\theta}\right)+\gamma I_{2}\right] i_{d q}+\right. \\
& \left.\frac{L_{s r}}{\sigma L_{s} L_{r}}\left[J n_{p} \dot{\theta}-\frac{1}{T_{r}} I_{2}\right] \lambda_{d q}+v_{d q}^{I D A}\right\}
\end{aligned}
$$

$v_{d q}^{I D A}$ is also here in charge to force currents to their desired values.

$$
v_{d q}^{I D A}=-R_{1} \tilde{i}_{d q}
$$

From this it comes that the Direct FOC is a particular case of the IDA-PBC of IM written in Disturbed-PCHD form.

\section{IDA-PBC OF VOLTAGE-FED IM WITH CURRENTS FEEDBACK}

Experimentally, the IM flux vector $\lambda$ is not measured. Only stator currents are measured. In this case the $d q$ frame is not appropriated since this frame depends on non-measured states (i.e. $\lambda_{r}$ ). So, it makes sense to design a control law that only needs stator currents feedback.
The IM electrical subsystem (13) can be rewritten in the following PCHD form :

$$
\begin{aligned}
{\left[\begin{array}{c}
\dot{\lambda}_{s} \\
\dot{\lambda}_{r}
\end{array}\right]=-\frac{1}{\sigma L_{s} L_{r}}[\begin{array}{c}
R_{s}^{2} L_{r} I_{2} \\
-R_{r} R_{s} L_{s r} e^{-J n_{p} \theta} \\
-R_{s} R_{r} L_{s r} e^{J n_{p} \theta} \\
R_{r}^{2} L_{s} I_{2}
\end{array} \underbrace{\left[\begin{array}{c}
R_{s}^{-1} \lambda_{s} \\
R_{r}^{-1} \lambda_{r}
\end{array}\right]}_{\nabla H}+\left[\begin{array}{l}
u \\
0
\end{array}\right]}
\end{aligned}
$$

where $H$ is the following energy-like function:

$$
\begin{gathered}
H(\lambda)=\frac{1}{2} \lambda^{T} R^{-1} \lambda \\
\Rightarrow \nabla H=\left[R_{s}^{-1} \lambda_{s} R_{r}^{-1} \lambda_{r}\right]^{T}
\end{gathered}
$$

The aim of the IDA-PBC method is to write the closed loop dynamics in the PCHD (or the Disturbed-PCHD) form as:

$$
\left[\begin{array}{c}
\dot{\tilde{\lambda}}_{s} \\
\dot{\tilde{\lambda}}_{r}
\end{array}\right] \equiv\left[\mathcal{J}_{d}(\tilde{x})-\mathcal{R}_{d}(\tilde{x})\right] \nabla H_{d}(\tilde{x})+\xi
$$

with $H_{d}(\tilde{\lambda})$ the desired closed loop energy-like function given by:

$$
H_{d}(\tilde{\lambda})=\frac{1}{2} \tilde{\lambda}^{T} R^{-1} \tilde{\lambda}
$$

Following the same procedure as in section (5.1), some straightforward calculation yields:

$$
\begin{aligned}
& {\left[\begin{array}{c}
\dot{\tilde{\lambda}}_{s} \\
\dot{\tilde{\lambda}}_{r}
\end{array}\right]=-\frac{1}{\sigma L_{s} L_{r}}\left[\begin{array}{c}
R_{s}^{2} L_{r} I_{2} \\
-R_{r} R_{s} L_{s r} e^{-J n_{p} \theta} \\
-R_{s} R_{r} L_{s r} e^{J n_{p} \theta} \\
R_{r}^{2} L_{s} I_{2}
\end{array}\right]\left[\begin{array}{c}
R_{s}^{-1} \tilde{\lambda}_{s} \\
R_{r}^{-1} \tilde{\lambda}_{r}
\end{array}\right]+\left[\begin{array}{c}
u-\gamma \\
0
\end{array}\right](40)}
\end{aligned}
$$

with:

$\gamma=\frac{1}{\sigma L_{s} L_{r}}\left(R_{s} L_{s r} e^{J n_{p} \theta} \lambda_{r}^{d}-R_{s} L_{r} \lambda_{s}^{d}\right)+\dot{\lambda}_{s}^{d}-2 R_{s} L_{s r} e^{J n_{p} \theta} \tilde{\lambda}_{r}$

Taking the control input as:

$u=\frac{1}{\sigma L_{s} L_{r}}\left(R_{s} L_{s r} e^{J n_{p} \theta} \lambda_{r}^{d}-R_{s} L_{r} \lambda_{s}^{d}\right)+\dot{\lambda}_{s}^{d}$

The following Disturbed-PCHD form is obtained

$$
\begin{aligned}
{\left[\begin{array}{c}
\dot{\tilde{\lambda}}_{s} \\
\tilde{\tilde{\lambda}}_{r}
\end{array}\right]=} & \underbrace{\frac{1}{\sigma L_{s} L_{r}}\left[\begin{array}{cc}
-R_{s}^{2} L_{r} I_{2} & -R_{s} R_{r} L_{s r} e^{J n_{p} \theta} \\
R_{r} R_{s} L_{s r} e^{-J n_{p} \theta} & -R_{r}^{2} L_{s} I_{2}
\end{array}\right]}_{\mathcal{J}_{d}(\tilde{x})-\mathcal{R}_{d}(\tilde{x})} \times \\
& {\left[\begin{array}{c}
R_{s}^{-1} \tilde{\lambda}_{s} \\
R_{r}^{-1} \tilde{\lambda}_{r}
\end{array}\right]+\underbrace{\left[\begin{array}{c}
2 R_{s} L_{s r} e^{J n_{p} \theta} \tilde{\lambda}_{r} \\
0
\end{array}\right]}_{\xi} }
\end{aligned}
$$

Then,

$\mathcal{J}_{d}(\tilde{x})=\frac{1}{\sigma L_{s} L_{r}}\left[\begin{array}{cc}0 & -R_{s} R_{r} L_{s r} e^{J n_{p} \theta} \\ R_{r} R_{s} L_{s r} e^{-J n_{p} \theta} & 0\end{array}\right]$ 


$$
\begin{gathered}
\mathcal{R}_{d}(\tilde{x})=\frac{1}{\sigma L_{s} L_{r}}\left[\begin{array}{cc}
R_{s}^{2} L_{r} I_{2} & 0 \\
0 & R_{r}^{2} L_{s} I_{2}
\end{array}\right] \\
\xi=\left[\begin{array}{c}
2 R_{s} L_{s r} e^{J n_{p} \theta} \tilde{\lambda}_{r} \\
0
\end{array}\right]=\underbrace{\left[\begin{array}{cc}
0 & 2 R_{s} R_{r} L_{s r} e^{J n_{p} \theta} \\
0 & 0
\end{array}\right]}_{A(\tilde{x})} \underbrace{\left[\begin{array}{c}
R_{s}^{-1} \tilde{\lambda}_{s} \\
R_{r}^{-1} \tilde{\lambda}_{r}
\end{array}\right]}_{\nabla H_{d}}
\end{gathered}
$$

Theorem 4. The equilibrium of the dynamic model (40) of the IM with the IDA-PBC control signal (41) is globally asymptotically stable.

Proof. The equilibrium of the dynamic model (40) of the IM with the control signal (41) is globally asymptotically stable if $\left[\mathcal{R}_{d}(\tilde{x})-A(\tilde{x})\right]$ is non negative (theorem 2).

$$
\begin{aligned}
& {\left[\mathcal{R}_{d}(\tilde{x})-A(\tilde{x})\right]=\left[\begin{array}{cc}
\frac{1}{\sigma L_{s} L_{r}} R_{s}^{2} L_{r} I_{2} & -2 R_{s} R_{r} L_{s r} e^{J n_{p} \theta} \\
0 & \frac{1}{\sigma L_{s} L_{r}} R_{r}^{2} L_{s} I_{2}
\end{array}\right]} \\
& {\left[\mathcal{R}_{d}(\tilde{x})-A(\tilde{x})\right] \geq 0 \Leftrightarrow \forall X \in \mathcal{R}^{4 \mathrm{x} 1}: \underbrace{X^{T}\left[\mathcal{R}_{d}(\tilde{x})-A(\tilde{x})\right] X}_{\triangleq F} \geq 0} \\
& F=X^{T}\left[\begin{array}{cc}
\frac{1}{\sigma L_{s} L_{r}} R_{s}^{2} L_{r} I_{2} & -2 R_{s} R_{r} L_{s r} e^{J n_{p} \theta} \\
0 & \frac{1}{\sigma L_{s} L_{r}} R_{r}^{2} L_{s} I_{2}
\end{array}\right] X \\
& =X^{T} \underbrace{\left[\begin{array}{cc}
\frac{1}{\sigma L_{s} L_{r}} R_{s}^{2} L_{r} I_{2} & -R_{s} R_{r} L_{s r} e^{J n_{p} \theta} \\
-R_{s} R_{r} L_{s r} e^{-J n_{p} \theta} & \frac{1}{\sigma L_{s} L_{r}} R_{r}^{2} L_{s} I_{2}
\end{array}\right]}_{\triangleq Q(\tilde{x})} X \\
& Q(\tilde{x})=\left[\begin{array}{cc}
\frac{1}{\sigma L_{s} L_{r}} R_{s}^{2} L_{r} I_{2} & -R_{s} R_{r} L_{s r} e^{J n_{p} \theta} \\
-R_{s} R_{r} L_{s r} e^{-J n_{p} \theta} & \frac{1}{\sigma L_{s} L_{r}} R_{r}^{2} L_{s} I_{2}
\end{array}\right] \\
& =\underbrace{\left[\begin{array}{cc}
R_{s} I_{2} & 0 \\
0 & R_{r} I_{2}
\end{array}\right]}_{R} \underbrace{\frac{1}{\sigma L_{s} L_{r}}\left[\begin{array}{cc}
L_{r} I_{2} & -L_{s r} e^{J n_{p} \theta} \\
-L_{s r} e^{-J n_{p} \theta} & L_{s} I_{2}
\end{array}\right]}_{L^{-1}(\theta)} \\
& \times \underbrace{\left[\begin{array}{cc}
R_{s} I_{2} & 0 \\
0 & R_{r} I_{2}
\end{array}\right]}_{R}
\end{aligned}
$$

It is clear that ${ }^{5} Q(\tilde{x})$ is positive definite $\Leftrightarrow L^{-1}(\theta)$ is positive definite, which is always verified. It can be concluded then, (According to theorem 2) that the dynamic model (35) of the IM with the IDAPBC control signal (41) is globally asymptotically stable.

Remark 3. If, as with (28), the control input (41) is modified such that:

$$
\begin{aligned}
u= & \frac{1}{\sigma L_{s} L_{r}}\left(R_{s} L_{s r} e^{J n_{p} \theta} \lambda_{r}^{d}-R_{s} L_{r} \lambda_{s}^{d}\right)+\dot{\lambda}_{s}^{d}+ \\
& 2 R_{s} L_{s r} e^{J n_{p} \theta} \tilde{\lambda}_{r}
\end{aligned}
$$

$5 Q \geq 0 \Leftrightarrow X^{T} Q X \geq 0 \Leftrightarrow \underbrace{X^{T} R}_{Z^{T}} L^{-1}(\theta) \underbrace{R X}_{Z} \geq 0 \Leftrightarrow Z^{T} L^{-1}(\theta) Z \geq 0$.
In this case, the closed loop is:

$$
\dot{\tilde{\lambda}}=\left[\mathcal{J}_{d}(\tilde{x})-\mathcal{R}_{d}(\tilde{x})\right] \nabla H_{d}
$$

Thus, the disturbance $\xi$ disappears but the cost is that the control (48) needs the measurement of the rotor flux $\lambda_{r}$ which is not available experimentally. This example illustrates the usefulness of theorem 2 that tolerates the existence of a disturbance (that is dominated by the controller) and consequently the controller is simpler and needs less measurements.

Remark 4. The Indirect FOC of the $\alpha \beta$ model of the IM given in Ortega et al. (1998) is:

$u=\underbrace{L_{s} \dot{\widehat{\hat{i}}}_{s d}+L_{s r} e^{J n_{p} \theta}{\dot{\widehat{i_{r d}}}}+n_{p} L_{s r} J e^{J n_{p} \theta} \dot{\theta} i_{r d}}_{\dot{\lambda}_{s}^{d}}+R_{s} i_{s d}(50)$

The control input given by (41) can be written as:

$u=\underbrace{\frac{1}{\sigma L_{s} L_{r}}\left(R_{s} L_{s r} e^{J n_{p} \theta} \lambda_{r}^{d}-R_{s} L_{r} \lambda_{s}^{d}\right)}_{R_{s} i_{s d}}+\dot{\lambda}_{s}^{d}=\dot{\lambda}_{s}^{d}+R_{s} i_{s d}(51)$

Thus, (51) and (50) are the same.

\section{CONCLUSION}

A large class of physical systems is addressed, the class of Disturbed-PCHD systems. A modified version of the IDA-PBC approach for PCHD systems is proposed: additional terms that can be external disturbances or considered as disturbances are tolerated. Two sufficient stability conditions are presented to deal with these disturbances. It is shown that this new approach can considerably simplify the controller and/or didn't need the measurement of all states. It is also shown that the controller can be robustified toward parameters uncertainties. The IDA-PBC of Disturbed-PCHD proposed here proves that the voltage-fed FOC of IM is included in the IDA-PBC of DisturbedPCHD systems.

\section{REFERENCES}

B.M. Maschke, R. Ortega, and A.J. van der Schaft. Energybased Lyapunov functions for forced Hamiltonian systems with dissipation. IEEE Conf. on Dec. and Contr., 1998.

B.M. Maschke, R. Ortega, A.J. van der Schaft, and G. Escobar. An energy-based derivation of lyapunov functions for forced systems with application to stabilizing control. Proc. 14th IFAC World Congress, Beijing, E: 409-414, 1999.

R. Ortega, A. Loria, P.J. Nicklasson, and H. SiraRamirez. Passivity-based control of Euler-Lagrange systems. Springer-Verlag, Berlin, 1998.

R. Ortega, A.J. van der Schaft, B. Maschke, and G. Escobar. Interconnection and damping assignment passivity-based control of port-controlled hamiltonian systems. Automatica, 38(4):585-596, 2002.

A.J. van der Schaft. $L_{2}-$ Gain and Passivity Techniques in Nonlinear Control. Springer-Verlag, Berlin, 2000.

A.J. van der Schaft and B. M. Maschke. On the hamiltonian formulation of nonholonomic mechanical systems. Reports on Mathematical Physics, 34(2):225-233, 1994. 